disruption of PPI mediated by 5-HT1A receptor stimulation. The action of haloperidol and raclopride suggests a major involvement of dopamine D2 receptors in this effect, possibly downstream from the initial serotonergic stimulation. The action of aripiprazole could be mediated by its partial agonist properties at 5-HT1A receptors or its dopamine D2 blocking properties.

\section{Longitudinal increases in gamma-phase synchrony contrasts with progressive gray matter atrophy in first-episode schizophrenia}

\section{T Whitford', D Alexander², J Brennan³ ', L Gomes ${ }^{3}$, E Gordon², A Harris ${ }^{4}$, L Williams ${ }^{1}$}

'Brain Dynamics Centre; ${ }^{2}$ Brain Resource International Database;

${ }^{3}$ Westmead Hospital; and ${ }^{4}$ The University of Sydney, Australia

Background: Our integrative neuroscience model of first-episode schizophrenia (FES) emphasizes a dysfunction in the coordinated neural activity required for selective attention in the disorder. This study investigated the longitudinal changes in neural connectivity (assessed by means of $40-\mathrm{Hz}$ gamma synchrony) and neuroanatomy [assessed by magnetic resonance imaging (MRI)] exhibited by patients with FES.

Method: Twenty-three FES patients underwent an EEG recording in response to an auditory oddball task, both at baseline and 2-3 years subsequently. Gammaphase synchrony was extracted from the EEG signal for $\mathrm{L} / \mathrm{R}$ frontal, temporal and posterior brain regions. Thirteen of these patients also underwent an MRI scan at baseline and follow-up, and an automated masking procedure was used to calculate the GM volumes of the analogous cortical regions. A $2 \times 6$ ('time' $\times$ 'region') repeated-measures ANOVA was used for statistical analysis.

Results: An inverse relationship was observed between the longitudinal changes in gamma synchrony and the longitudinal changes in GM volume. While the patients with FES lost significant frontal and parietal GM over the follow-up interval, they also showed a corresponding increase in posterior gamma-phase synchrony.

Conclusions: These results indicate that while gammaphase synchrony increases over the initial years of illness in patients with FES, GM volume decreases in corresponding cortical regions. Given the role that gamma-phase synchrony has been proposed to play in the integration of discrete perceptual events, these findings support the idea that schizophrenia is caused by a dysfunction in neural connectivity.

\section{Late-onset bipolar disorder: preliminary results from Sydney}

\author{
C Wijeratne', A Olley', G Mahli' ${ }^{2,3,4}$, M Philip'
}

'School of Psychiatry, University of New South Wales, Sydney, Australia; ${ }^{2}$ Academic Discipline of Psychological Medicine, Northern Clinical School, University of Sydney, New South Wales, Australia; ${ }^{3}$ Black Dog Institute, Sydney, Australia; " Prince of Wales Medical Research Institute, Sydney, Australia

Background: Previous studies have suggested that there may be bipolar disorder subtypes according to age at onset (AAO), including a late-onset (LO) group with onset in the fifth decade of life. LO presentations may be associated with greater cerebrovascular disease and increased neuropsychological deficits. Different AAO may also explain some of the genetic heterogeneity associated with bipolar disorder.

Method: We have commenced recruitment of participants aged 40 years and over, with the aim of assessing early-onset bipolar I, late-onset bipolar I and healthy control groups. Assessment tools included the following: sociodemographic and disability questionnaires, SCID, HDRS, YMRS; cerebral magnetic resonance imaging scan; a neuropsychological battery and venepuncture for genetic testing.

Results: Preliminary results for the first 15 participants with bipolar disorder (mean age 53.9 years, range 46-66 years, 66\% women) have shown an average latency of 11 years between the first affective episode and the first episode of mania, and of 17 years before a formal diagnosis of bipolar disorder. There was a high rate of comorbidity with anxiety disorders. Contrary to study hypotheses, the participants tended to be relatively physically healthy with minimal vascular disease burden. Neuropsychological assessment of euthymic participants showed no differences in language and memory, but significant differences in visuospatial organization and selfmonitoring tasks.

Conclusions: These preliminary results suggest deficits in frontal executive dysfunction in this sample of older bipolar participants. The recruitment of a relatively young and ambulatory sample may have led to the finding of minimal vascular disease.

\section{SSRI use and bone mineral density in women with a history of depression: Geelong Osteoporosis Study}

\section{Williams, M Henry, M Berk, S Dodd, F Jacka, M Kotowicz, G Nicholson, J Pasco}

The University of Melbourne, Melbourne, Australia

Background: Selective serotonin reuptake inhibitors (SSRIs) are a first-line treatment for depression. 
SSRIs have been reported to regulate serotonin (5-HT) receptors and the transporter 5-HTT on osteoclasts and osteoblasts. Previous studies reporting reduced bone mineral density (BMD) among SSRI users may have been confounded by the effects of depression.

Methods: Among women enrolled in the Geelong Osteoporosis Study (GOS), a history of depression was ascertained by clinical interview (SCID-I/NP). BMD was measured at the PA spine, hip, total body and forearm using dual-energy absorptiometry, and medication use was self-reported.

Results: Among 177 women with a lifetime history of depression, current users of bisphosphonates, glucocorticoids, hormone therapy and other antidepressants were excluded $(n=49)$. Of the remaining 128 (median age 51.5 years, range 30-74), $26(20.3 \%)$ reported current SSRI use. SSRI users were shorter than nonusers $(1.59 \pm 0.06$ vs. $1.62 \pm 0.06 \mathrm{~m}$, $P=0.01$ ); however, there were no differences in age, weight or smoking history. Using analysis of covariance and controlling for age, weight, height and smoking history, BMD among SSRI users was 5.7\% lower at the femoral neck $(0.977 \pm 0.015$ vs. $0.922 \pm$ $\left.0.025 \mathrm{~g} / \mathrm{cm}^{2}, P=0.03\right), 6.1 \%$ lower at the trochanter $\left(0.813 \pm 0.010\right.$ vs. $\left.0.763 \pm 0.021 \mathrm{~g} / \mathrm{cm}^{2}, P=0.04\right)$ and $4.4 \%$ lower at the midforearm $(0.745 \pm 0.009$ vs. $\left.0.712 \pm 0.015 \mathrm{~g} / \mathrm{cm}^{2}, P=0.03\right)$ than nonusers. No differences in BMD were detected at other sites.

Conclusions: Among women with a lifetime history of depression, SSRI use is associated with reduced BMD. Although the mechanism remains unclear, these observations are consistent with a role for the serotonergic system in regulating bone metabolism.

\section{'Gift of Hope' - motivation for brain donation into schizophrenia research}

\section{R Wilson', P Terwee', T Garrick ${ }^{1,3}$, C Harper ${ }^{3}$}

'Neuroscience Institute of Schizophrenia and Allied Disorders (NISAD); ${ }^{2}$ The

Rhumbline Group; and ${ }^{3}$ Discipline of Pathology, University of Sydney, Sydney, Australia

Background: Schizophrenia is an illness that is unique to humans and animal models are of limited use. Consequently, NISAD has established a brain tissue bank and an associated premortem donor program called the Gift of Hope (GOH). This program invites people aged 18 years and over to donate their brain (after death) for research into Schizophrenia. The main benefit of $\mathrm{GOH}$ is that it provides researchers with high-quality, well-characterized tissues. This study is designed to identify why individuals are motivated to become donors in such a program.

Methods: Participants from the GOH database, who have a Sydney metro postal address, were selected to receive the paper-based, 26-item questionnaire that was developed by the 'Using Our Brains' group. It consisted of both open-ended and fixed responses designed to collect demographic information and the participant's comments on their reasons for donation.

Results: Forty-five participants completed and returned the questionnaire, a response rate of $60 \%(n=74)$. Personal experience of the illness was reported by $50 \%$ of the participants as their main reason for donating. A further $22 \%$ donated in the hope of improving knowledge and research in the area and $17 \%$ donated for altruistic reasons.

Conclusions: These preliminary results suggest that personal experience of schizophrenia, which includes being a family member of someone with the illness, is the key motivating factor when it comes to brain donation for the $\mathrm{GOH}$ program. 